\title{
A CORRELATIVE STUDY OF FNAC THYROID WITH THYROID HORMONE PROFILE
}

Ritica Chaudhary ${ }^{1}$, Zulfikar Ahmed ${ }^{2}$, Umaru N³

\section{HOW TO CITE THIS ARTICLE:}

Ritica Chaudhary, Zulfikar Ahmed, Umaru N. "A Correlative Study of FNAC Thyroid with Thyroid Hormone Profile". Journal of Evolution of Medical and Dental Sciences 2014; Vol. 3, Issue 06, February 10; Page: 1474 1480, DOI: $10.14260 /$ jemds $/ 2014 / 2010$

ABSTRACT: BACKGROUND: Study aims to determine correlation of the cytological pattern of thyroid lesions in addition to the utility and accuracy of FNAC as initial diagnostic method along with thyroid hormone profile (T3, T4 and TSH) which provides information at physiological and pathological levels and thus can enhance the likelihood of early detection of ambiguous thyroid dysfunction with minimal clinical findings. OBJECTIVES: To obtain role of thyroid hormone profile (T3, T4 and TSH) aimed at defining prognostic parameters and correlating it with cytology results, diagnosis of diffuse nontoxic goiter, diagnosis of solitary/dominant thyroid nodule, confirmation of clinically obvious thyroid malignancy. MATERIALS AND METHODS: 150 patients with thyroid swellings underwent FNAC along with complete thyroid hormone profile in Central Diagnostic Lab of AJIMS Mangalore. Procedure was performed without L.A with the help of aspirating technique using 23G needle attached to $10 \mathrm{ml}$ disposable syringe. An average of one attempt per nodule was performed usually resulting in 4 direct smears. Both air dried and wet fixed smears fixed in $95 \%$ alcohol for about 30 minutes and stained with Leishman's stain and Pap stain examined under light microscope. Thyroid profile is also performed in all the above patients. RESULTS: One hundred and fifty aspirations of patients (both females and males) were analysed.T3, T4 and TSH was analyzed for all 150 patients. The mean age of patients in this study was 39.66 years and age range was 1-76 years. There were 142 females (94.66\%) and 8 males (5.33\%). Maximum number of patients was in the age range 21-40 years (49.3\%). Most common lesion was colloid goitre accounting for 98 number of cases. There were 141 non-neoplastic lesions, 9 cases were found to be neoplastic. Maximum number of patients, 92 cases was euthyroid while minimum numbers of patients, 4 cases were found to be hypothyroid. 17 cases were hyperthyroid, 23 cases were subclinical hyperthyroidism while 14 cases were subclinical hypothyroidism respectively. CONCLUSION: It renders unnecessary the need for excisional biopsy in advanced disease, elderly patients or in case where treatment is non-surgical. All the fine needle aspiration diagnosis must be viewed in the light of the clinical picture and thyroid hormone profile to minimize the risks of a false-negative report. The widespread application of this method should be fully encouraged as it fulfills a genuine medical need. FNAC is gold standard for preoperative assessment of thyroid nodule. Early and accurate diagnosis reduces surgical intervention, morbidity and mortality.

KEYWORDS: FNAC THYROID, T3, T4, TSH.

INTRODUCTION: Thyroid fine needle aspiration cytology is over 50 years old ${ }^{1}$ and is the principal method of preoperative diagnosis in both children and adults. It has been shown to be superior to clinical, radionucleotide or thyroid ultrasound assessment alone. FNAC requires careful aspiration technique and interpretation of the cytological findings. Most practitioners rely on FNAC alone, especially for the first attempt at diagnosis. Core biopsy histological assessment of the 
thyroid is described in the literature ${ }^{2}$ and core biopsy cytological assessment of the thyroid is used in some centres. ${ }^{3}$ Thyroid nodules are very frequent, with a number of studies showing an annual incidence rate of $4-8 \% .{ }^{4}$ Autopsy and ultrasound data suggests that the prevalence rate for thyroid nodules in clinically normal individuals is around $50 \% .^{5}$

Three to sixteen percent of patients who undergo thyroidectomy for benign disease are found to have incidental malignancies, mostly small papillary carcinomas less than $10 \mathrm{~mm}$ in size, the majority of which are of little significance. ${ }^{6}$ Despite the high prevalence of thyroid nodules and incidental malignancies, the prevalence of clinically overt thyroid carcinoma is much lower. ${ }^{7}$

Its use has decreased the number of thyroid surgeries performed and increased the ratio of malignant to benign lesions resected. As a result many thyroid surgeries for non-neoplastic diseases have been avoided.

Thyroid swellings are common occurrence in most regions of the world. India has the world's biggest goiter belt in sub-Himalayan region. FNAC is a well- established OPD procedure used in primary diagnosis of thyroid swellings. FNAC by giving direct morphological information often bridges the gap between clinical findings and laboratory tests to give a definite diagnosis and subsequently reduced the need of surgery. Thyroid nodules are more common in women, and the incidence increases with age, a history of radiation exposure, and a diet containing goitrogenic material. The vast majority of these nodules are non-neoplastic lesions .However, the distinction of these benign lesions from a malignancy cannot be based reliably on the clinical presentation only. Several diagnostic tests, such as radionuclide scanning, high-resolution ultrasonography, and fineneedle aspiration biopsy have been used to select the patient population requiring surgical intervention. Recent studies have demonstrated that among all these diagnostic modalities, FNA is the most accurate, cost-effective, and simplest screening test for rapid diagnosis of thyroid nodules. Fine-needle aspiration has also been shown to have similar or higher sensitivity and accuracy levels than frozen section examination

MATERIALS AND METHODS: This present study was conducted at the Department of Pathology, A.J Institute of Medical Sciences, Mangalore. Fine needle aspirations of thyroid lesions from 150 patients (both males and females) formed the material for this study. The duration of the study was from May 2011 to May 2013.

The clinical details included the patient's particulars, clinical history and relevant investigations. The aspirations were performed on any thyroid abnormality observed by physical examination or complained by the patients.

The cytology accession number was given to each fine needle aspiration. Sampling was performed without any local anesthesia. Skin over the swelling was cleaned with spirit.

The technique for aspiration: The needle attached to a $5 \mathrm{ml}$ disposable plastic syringe inserted in a syringe holder. The syringe and the gun are manipulated with the dominant hand while the biopsy site is stabilized with the other hand. The needle is introduced briskly perpendicular to the swelling without anesthesia. The small lesions are aspirated from the center while the larger ones are peripherally approached.

If the mass is cystic, the cyst fluid is collected in the bottles for the preparation of smears after centrifugation. The smears were either air-dried or fixed in alcohol (95\%). Air dried smears 
were stained by the MGG stain while alcohol fixed specimens were stained by the papanicolauo method.

RESULTS: The mean age of patients in this study is 39.66 years and age range is 1-76 Years (table 1). In this study total number of females was 142 (94.6\%) while males were $8(5.4 \%)$.

\begin{tabular}{|c|c|}
\hline Age & Number of patients \\
\hline 0-20yrs & 9 \\
\hline 21-40yrs & 74 \\
\hline 41-60yrs & 57 \\
\hline 61-80yrs & 10 \\
\hline TABLE 1: DISTRIBUTION OF THYROID \\
LESIONS ACCORDING TO AGE \\
\hline
\end{tabular}

9 patients were in the age range of 0-20 years, 57 patients were in the age range 41-60 years, 10 patients were in the age range of 61-80 years. Maximum number of patients was in the age range 21 to 40 years, 74 cases while least number of patients was in the age range of 0-20 years. Out of 150 patients maximum numbers of patients were diagnosed with colloid goiter (Figure 1) with 98cases. There were 7 malignant cases and 2 follicular neoplasms which were found out to be follicular adenoma histopathologically. There were 4 cases of colloid cyst while one case of thyroglossal cyst. Out of 30 cases of thyroiditis, 9 were lymphocytic thyroiditis (Figure 2), 18 were Hashimoto's thyroiditis while 3 were de quervian's thyroiditis. 8 cases were primary hyperplasia. In this study out of 150 cases, 9 cases were neoplastic and rest were non- neoplastic (Table2). The neoplastic cases distribution was as follows, 3 cases were metastatic to the thyroid, 2 were papillary carcinoma (Figure3), and the rest 2 were anaplastic carcinoma and medullary carcinoma (Figure 4) respectively. 2 cases were follicular neoplasms which were found to be follicular adenoma on histopathology.

\begin{tabular}{|c|c|}
\hline CYTOLOGICAL DIAGNOSIS & Number of cases \\
\hline GOITRE & 98 \\
\hline COLLOID CYST & 4 \\
\hline THYROGLOSSAL CYST & 1 \\
\hline HYPERPLASIA & 8 \\
\hline DEQUERVIAN'S THYROIDITIS & 3 \\
\hline HASHIMOTO'S THYROIDITIS & 18 \\
\hline LYMPHOCYTIC THYROIDITIS & 9 \\
\hline FOLLICULAR NEOPLASM & 2 \\
\hline METASTASIS & 3 \\
\hline ANAPLASTIC CARCINOMA & 1 \\
\hline MEDULLARY CARCINOMA & 1 \\
\hline PAPILLARY CARCINOMA & 2 \\
\hline $\begin{array}{c}\text { TOTAL } \\
\end{array}$ & 150 \\
\hline
\end{tabular}




\section{STATUS}

\begin{tabular}{|l|c|}
\hline \multicolumn{1}{|c|}{ HORMONAL STATUS } & NUMBER OF PATIENTS \\
\hline EUTHYROID & $92(61.3 \%)$ \\
\hline HYPERTHYROID & $17(11.3 \%)$ \\
\hline HYPOTHYROID & $4(2.6 \%)$ \\
\hline SUBCLINICAL HYPERTHYROIDISM & $23(15.3 \%)$ \\
\hline SUBCLINICAL HYPOTHYROIDISM & $14(9.3 \%)$ \\
\hline TOTAL & 150 \\
\hline \multicolumn{2}{|l|}{ TABLE 3: DISTRIBUTION OF CASES ACCORDING TO HORMONE } \\
\hline
\end{tabular}

Out of 150 cases maximum number of patients, 92 cases were euthyroid while minimum number of patients, 4 cases was found to be hypothyroid. 17 cases were hyperthyroid, 23 cases were subclinical hyperthyroidism while 14 cases were of subclinical hypothyroidism respectively (Table3). Thyroid hormone status distribution in non-neoplastic cases was as follows: Out of 141 cases $61 \%$ were euthyroid, $12 \%$ were hyperthyroid, $2.8 \%$ were hypothyroid while $15.6 \%$ and $8 \%$ were subclinical hyperthyroidism and subclinical hypothyroidism respectively.

Out of total 9neoplastic lesions, 6 cases were euthyroid. One case of papillary carcinoma and 1 case of follicular neoplasm was found to be subclinical hypothyroidism and 1 case of anaplastic carcinoma was found to be subclinical hyperthyroidism. In lymphocytic thyroiditis, out of 9 cases, 7 cases were euthyroid, 1 was hyperthyroid and 1 was subclinical hypothyroid. In Hashimoto's thyroiditis out of 18 cases, 11 cases were euthyroid, 4 were subclinical hypothyroid, 2 were subclinical hyperthyroid and 1 case was hypothyroid. In de quervian's thyroiditis out of 3 cases 2 were hyperthyroid while 1 was found to be euthyroid. All 4 cases of colloid cyst were euthyroid while one case of thyroglossal cyst was also euthyroid. Out of 8 cases of primary hyperplasia 4 were hyperthyroid, 2 were euthyroid while 2 were subclinical hyperthyroid. Out of 98 cases of colloid goiter, 60 cases were euthyroid, 18 were subclinical hyperthyroid, 10 were hyperthyroid while 7 were subclinical hypothyroid and remaining 3 were hypothyroid.

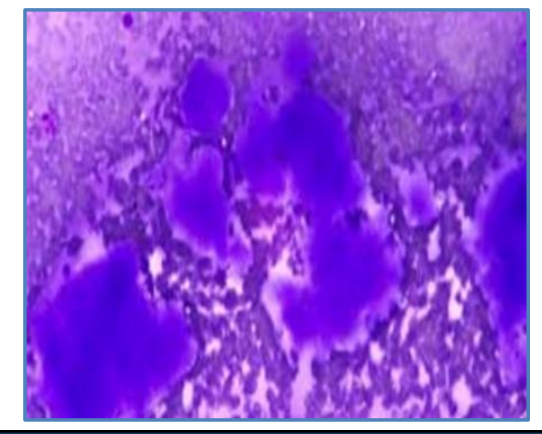

Fig. 1: Abundant thick colloid in Colloid Goitre (10x)

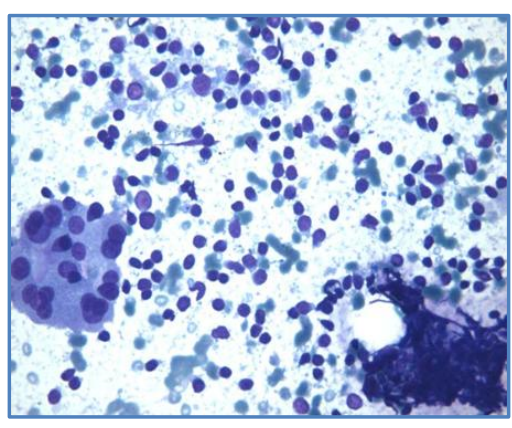

Fig. 2: Microphotograph showing lymphocytic thyroiditis(40x) 


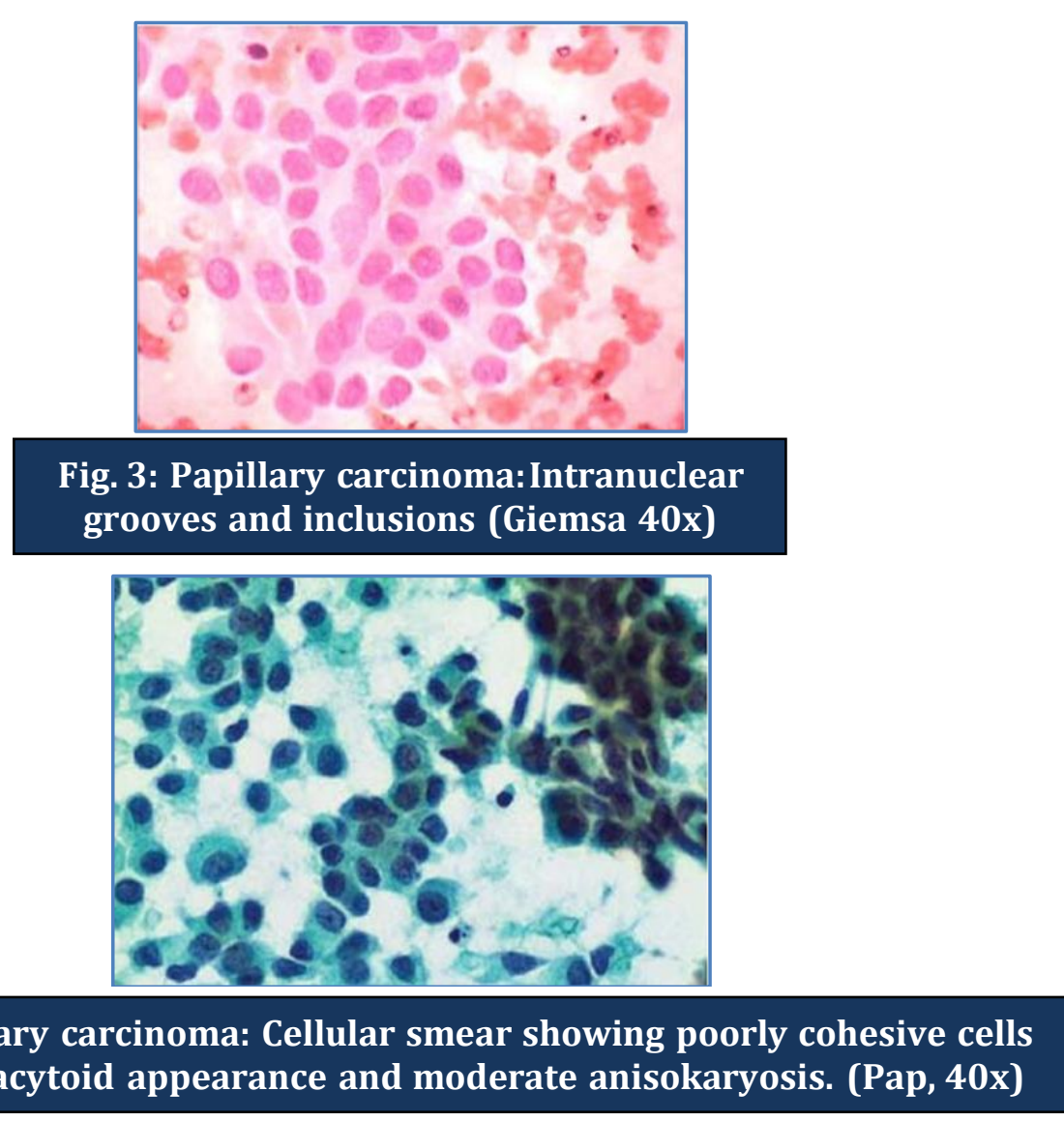

DISCUSSION: Thyroid nodules are very common occurring in $4 \%$ of the population aged between 30 and $60 . .^{8}$ Most are benign and only between $10 \%$ and $20 \%$ are malignant. 9,10 Therefore, surgery as the initial intervention or investigation will have a very low yield. Very few diagnostic tests help differentiate between benign and malignant nodules. Thyroid ultrasound can distinguish solid from cystic lesions but not all cystic lesions are benign. ${ }^{11}$ Thyroid isotope scans usingtechnetium-99 classify nodules as hot or cold. Hot nodules are functioning and should be benign whereas cold nodules are non-functioning and might be malignant. However, fewer than $20 \%$ of cold nodules are malignant leading to a high false positive rate.

The addition of thallium scans is a newer development but again has high false positive rates. The poor accuracy and high cost of nuclear imaging plus the significant radiation burden it places on patients has led some to suggest that its routine use in investigating the solitary thyroid nodules should be abandoned, while recognizing its value in follow up after a thyroid malignancy.12,13 FNA cytology is inexpensive, can be performed in an outpatients clinic, and has few complications including absence of tumor implants along the needle track. ${ }^{14}$ Inadequate biopsies are said to be less common when the pathologist reporting the biopsy does the aspiration personally.

In the present study age of the patients ranged from 1-76 years, with a median age of 39.66. Age distribution and median age of present study was comparable to S. Mahar et al (2006) study and S. Chandanwale ${ }^{15}$ et al (2012) study but the median age was lower when compared to studies by Burch et al (1996). Out of 150 patients majority were females, 142 (95\%) and 8 males 
(5\%). Sex distribution was similar when compared to studies by Silverman ${ }^{16}$ et al (1986) and Leonard et al (1997). In the present study distribution of neoplastic and non-neoplastic lesions were $6 \%$ and $94 \%$ respectively which was similar when compared to study done by S. Chandanwale et al (2012). Out of 150 patients, 98(65.3\%) presented with colloid goiter. Colloid goiter presented with various changes like cystic degeneration, focal hyperplasia and has been included in the study. It was the most common lesion and it was in accordance with the study done by S. Chandanwale et al (2012). In the present study the highest percentage of neoplastic lesion was metastatic while in studies done by S. Mahar et al (2000), S. Chandanwale et al (2012) and Tabaqchali ${ }^{17}$ et al (2006), the highest percentage of neoplastic lesion was papillary carcinoma of thyroid.

2 cases of follicular neoplasms diagnosed cytologically were found to be follicular adenomas histologically. In this study 3 cases out of 7 malignant lesions were metastatic cancers to thyroid. Primary cancer was adenocarcinoma colon, adenocarcinoma pancreas and MPNST respectively which is rare. In the study done by E. Mirallie et al (2005), twenty-nine patients (41 to 78 years) had intrathyroid metastases. Primary cancers were renal cell in 16 patients, lung in 4 , digestive in 4, sarcoma in 1, melanoma in 1, neuroendocrine in 1, and of unknown origin in 1 patient. In another study done by K. Wood et al (2013), kidney was the most common primary site of origin, 4 out of 15 patients had renal cell carcinoma as primary tumor. Metastatic malignancy to the thyroid gland, although considered rare, occurs more frequently than expected. Microscopic metastases to the thyroid gland have been reported to occur in 4\%-9\% of autopsy studies with breast, lung, melanoma and kidney the most common primary malignancies. Maximum number of patients (61.3\%), both neoplastic and non-neoplastic cases in this study was euthyroid. 50\% of the cases of primary hyperplasia were hyperthyroid and $25 \%$ were subclinical hyperthyroid hence found to be correlating with the FNA diagnosis.

CONCLUSION: Fine needle aspiration cytology is an easy technique of obtaining material for examination, with little discomfort to the patient. The rapidity, reliability, reproducibility and low cost of the method are main merits. It helps in the rapid diagnosis of neoplastic, hyperplastic and inflammatory lesions. When a malignancy is identified, it permits the psychological preparation of the patient before definitive surgery. It renders unnecessary the need for excisional biopsy in advanced disease, elderly patients or in case where treatment is non-surgical. All the fine needle aspiration diagnosis must be viewed in the light of the clinical picture and thyroid hormone profile to minimize the risks of a false-negative report. The widespread application of this method should be fully encouraged as it fulfills a genuine medical need. FNAC is gold standard for preoperative assessment of thyroid nodule. Early and accurate diagnosis reduces surgical intervention, morbidity and mortality.

\section{REFERENCES:}

1. Soderstrom N. Puncture of goitres for aspiration biopsy. Acta Med Scand 1952; 144: 237-44.

2. Screaton NJ, Berman LH, Grant JW. US guided core needle biopsy of the thyroid gland. Radiology2003; 226:827-32.

3. Mehrotra P, Viswanathan H, Johnson SJ et al. Ultrasound guidance improves the adequacy of our preoperative cytology but not its accuracy. Cytopathology 2006; 17:137-44. 


\section{ORIGINAL ARTICLE}

4. Cramer H. Fine needle aspiration cytology of the thyroid: an appraisal. Cancer Cytopathol2000; 90:325-9.

5. American Association of Clinical Endocrinologists and Associazione Medici Endocrinologi medical guidelines for clinical practice for the diagnosis and management of thyroid nodules. Endocr Pract 2006; 12:63-103.

6. Miccoli P, Minuto MN, Galleri D et al. Incidental thyroid carcinoma in a large series of consecutive patients operated on for benign thyroid disease. ANZ J Surg2006; 76: 123-6

7. Poller D.N, Stelow E.B, Yiangou C. Thyroid FNAC cytology: can we do it better? Cytopathlology 2007; $1-2$.

8. Rosen IR et al .Ultrasound -guided fine needle aspiration biopsy in management of thyroid disease. Am J Surg 1993; 166: 346-9.

9. Franklyn JA et al. The value of imaging in the diagnosis of thyroid cancer. Nuclear Med Comm 1992;13: 641-3.

10. Choo C. Cost effectiveness of nuclear medicine. Lancet 1995; 345:512.

11. Mandrekar SRS et al, Role of fine needle aspiration as the initial modality in investigation of thyroid lesions. Acta Cytol 1995; 39: 898-903.

12. Mazzaferi EL et al. Management of a solitary thyroid nodule. Eng J Med 1993; 328: 553-9.

13. Blanco CC et al. Diagnostic efficacy of fine needle aspiration biopsy in patients with thyroid nodular disease. Analysis of 510 cases. Rev Clin Esp.2005; 205: 374-8.

14. Leonard N, Melcher DH. To operate or not to operate? The value of fine needle aspiration cytology in assessment of thyroid swellings. Jc Clin Pathol 1997;941-943.

15. Chandanwale S. et al. Clinicopathological correlation of thyroid nodules. Int J Pharm Biomed 2012; 97-102.

16. Silverman et al. The role of fine needle aspiration biopsy in the rapid diagnosis and management of thyroid neoplasm. Cancer 1986; 57: 164-70.

17. Tabaqchali MA et al. Thyroid aspiration cytology in Newcastle. Am R Coll Surg Engl 2000; 82: 149-155.

\section{AUTHORS:}

1. Ritica Chaudhary

2. Zulfikar Ahmed

3. Umaru N.

\section{PARTICULARS OF CONTRIBUTORS:}

1. Post Graduate, Department of Pathology, A. J. Institute of Medical Sciences, Mangalore.

2. Associate Professor, Department of Pathology, A. J. Institute of Medical Sciences, Mangalore.

3. Professor and HOD, Department of Pathology, A. J. Institute of Medical Sciences, Mangalore.

\section{NAME ADDRESS EMAIL ID OF THE} CORRESPONDING AUTHOR:

Dr. Zulfikar Ahmed, Associate Professor, Department of Pathology, A. J. Institute of Medical Sciences, Mangalore. E-mail: zulfikaraha@gmail.com

Date of Submission: 09/01/2014. Date of Peer Review: 10/01/2014. Date of Acceptance: 28/01/2014. Date of Publishing: 06/02/2014. 